\title{
Elderly Obesity: Is It Harmful or Beneficial?
}

\author{
Tae Nyun Kim* \\ Cardiovascular and Metabolic Disease Center, Inje University College of Medicine, Busan; Division of Endocrinology and Metabolism, Department of Internal \\ Medicine, Inje University Haeundae Paik Hospital, Busan, Korea
}

The prevalence of obesity among elderly populations is a matter of increasing concern. Elderly obesity is a pathophysiologically complex issue, and predictions regarding obesity-related comorbidity and weight management are challenging. As individuals age, changes in the body composition, such as increase in fat mass and decrease in muscle mass, are observed even in the absence of changes in body weight and body mass index. Hence, body mass index and other indices of obesity should be cautiously interpreted in the elderly, and weight loss should be recommended for obese elderly individuals with functional limitations or metabolic complications that may improve with weight loss. By the year 2026, more than $20 \%$ of the Korean population will be aged 65 years or older, and this along with the obesity epidemic is expected to pose an increasingly serious financial problem for the Korean healthcare system. This review summarizes the epidemiology of obesity in Korea, the clinical implications of elderly obesity, and considerations regarding weight management. Understanding the complexity of elderly obesity could facilitate the design of preventative and therapeutic strategies that would have a positive impact on the overall health of the Korean population.

Key words: Obesity, Sarcopenia, Sarcopenic obesity, Epidemiology, Aged

\author{
Received May 22, 2018 \\ Reviewed June 7, 2018 \\ Accepted June 18, 2018 \\ *Corresponding author \\ Tae Nyun Kim
}

https://orcid.org/0000-0001-6568-2469

Division of Endocrinology and Metabolism, Department of Internal Medicine, Inje University Haeundae Paik Hospital, 875 Haeun-daero, Haeundae-gu, Busan 48108, Korea Tel: +82-51-797-0661

Fax: +82-51-797-2070

E-mail: kimtn031@gmail.com

\section{INTRODUCTION}

Progressive aging of the population and the steady increase in life expectancies in prosperous countries are paralleled by increases in age-associated metabolic diseases and functional limitations. ${ }^{1,2}$ Korea is not an exception to this trend and is expected to become a super-aged society by 2026 , with more than $20 \%$ of the population older than 65 years of age. In a recent study, researchers predicted that the average life expectancy of women in Korea will exceed 90 years by $2030^{3}$, and Korean men are projected to make similarly large gains. However, this increase in life expectancy does not necessarily correspond to an increase in healthy life years.

In addition to population aging, obesity is a complex and increasingly prevalent condition that affects the quality of life, increases the risk of illness, and reduces the disability-adjusted life expectan- cy in both elderly and young populations worldwide. ${ }^{4,5}$ According to the Asia-Pacific criteria of the World Health Organization guidelines, body mass index (BMI) values of $23.0-24.9 \mathrm{~kg} / \mathrm{m}^{2}, 25.0$ $29.9 \mathrm{~kg} / \mathrm{m}^{2}$, and $\geq 30 \mathrm{~kg} / \mathrm{m}^{2}$ correspond to the classifications of overweight, grade I obesity, and grade II obesity, respectively, regardless of age. ${ }^{6}$ In addition, abdominal obesity is defined by the Korean Society for the Study of Obesity as a waist circumference $>90 \mathrm{~cm}$ in men and $>85 \mathrm{~cm}$ in women. In Korea and many other countries, increases in the prevalence of obesity have been observed in all age groups, with steady and significant increases in prevalence of obesity and abdominal obesity among the elderly. ${ }^{6}$ The aging population and obesity epidemic together signify a double disease burden for the future. These epidemiological trends represent an increasingly serious financial problem that will be faced by the Korean healthcare system. 
Obesity is a more pathophysiologically complex condition in older people than in young and middle-aged adults. This complexity makes it difficult to predict obesity-related comorbidities and presents a clinical conundrum in terms of weight management. BMI-defined obesity is strongly associated with cardiometabolic risk factors, and the use of BMI to classify obesity is simple and convenient from a treatment perspective. However, BMI does not account for variations in lean body mass, fat mass, or fluid retention. ${ }^{7}$ Moreover, aging is often accompanied by progressive loss of muscle mass and strength (i.e., sarcopenia) and an increase in fat mass, even in individuals with relatively stable BMI. ${ }^{8,9}$ Accordingly, it remains uncertain whether BMI is an appropriate measure of obesity in older individuals. Instead, parameters such as waist circumference and measurement of body composition may be more important than BMI in this population. In addition, weight management in elderly people should aim to improve and maintain physical functioning and quality of life, as well as prevent medical problems associated with obesity in young and middle-aged patients. $^{10}$

Elderly obesity is a multifactorial condition resulting from longterm imbalance between energy intake and expenditure, with is influenced by genetic, epigenetic, and environmental factors. ${ }^{11,12}$ In addition, sociocultural factors may play a role in the national prevalence of obesity. In developing countries, overweight and obesity tend to be more prevalent among women, while in developed countries, obesity is more common among men. ${ }^{4}$ In accordance with this trend, the prevalence of abdominal obesity and general obesity in Korea is more rapidly increasing among men than among women ${ }^{6}$, and various factors have been suggested to contribute to age- and sex-related differences in obesity patterns. This paper reviews these differences in terms of the epidemiology of obesity in Korea, and discusses the clinical implications of obesity and related considerations regarding weight management in the elderly.

\section{PREVALENCE OF OBESITY, SARCOPENIA, AND SARCOPENIC OBESITY IN THE ELDERLY}

The prevalence of obesity continues to increase among all age groups. ${ }^{6}$ In Korea, the prevalence rates of general obesity and abdominal obesity peak at approximately 60 and 70 years, respectively, after which point body weight stabilizes and begins to decline. However, current trends indicate that the prevalence of obesity will continue to increase among the elderly. According to the National Health Insurance Service database, the frequency of obesity among adults aged $70-79$ years increased from $31.7 \%$ in 2006 to $36.6 \%$ in 2015 , whereas among those aged $\geq 80$ years, the frequency of obesity increased from $21.9 \%$ in 2006 to $27.5 \%$ in 2015 . Similar trends were observed for the prevalence of abdominal obesity among the elderly, as well as the presence of obesity among young adults (ages 20-29 years, 30-39 years, and 40-49 years). ${ }^{6}$

From 2014 to 2015, the prevalence of BMI-defined obesity among men increased up to the age of 30 years and then decreased from 40 to 50 years of age. ${ }^{6}$ In women, however, it increased until the mid-70s and decreased thereafter. In contrast, the prevalence of abdominal obesity steadily increased from 20-30 years of age to 70 years of age in both men and women, followed by decreases among those older than 80 years. Interestingly, the prevalence of abdominal obesity in those younger than 60-70 years of age was higher among men than women, whereas the reverse was true thereafter. The evidence clearly suggests sex-based differences in body composition and prevalence of obesity.

Fig. 1 presents age-related trends in appendicular skeletal muscle mass and visceral fat area, based on data from the Korean Sarcopenic Obesity Study (KSOS). ${ }^{13}$ Although young and middle-aged men had more visceral fat than women of the same age, the total incremental change in visceral fat area with aging was greater in women than in men. In men, the appendicular skeletal muscle mass increased with age, peaked at approximately $20-30$ years, and showed a dramatic decrease thereafter. However, in women, considerably different changes in muscle mass were observed with aging. These results were consistent with the analyses of data from the Korea National Health and Nutrition Examination Survey 2008-2010, which showed that, in women, muscle mass slowly increased until approximately 40 years of age, remained constant until 50-60 years of age, and decreased slightly thereafter. ${ }^{14}$ Hence, in the Korean elderly population, the prevalence of class II sarcopenia is higher among men than among women when using the unadjusted or height-adjusted appendicular skeletal muscle mass al- 

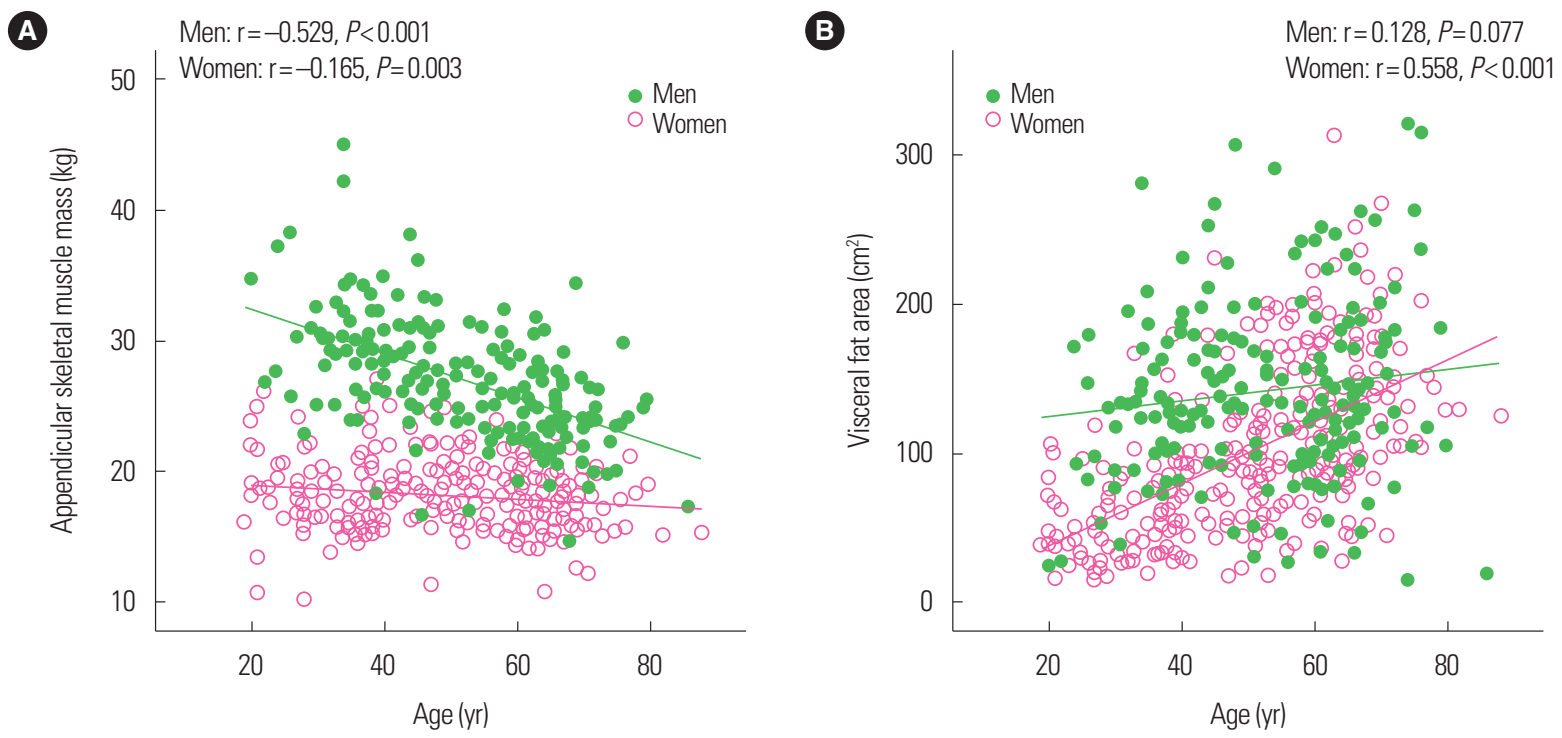

Figure 1. Relationships of age with appendicular skeletal muscle mass (A) and visceral fat area (B) in both men and women (based on data from the Korean Sarcopenic Obesity Study)..$^{13}$

though the prevalence varies among operating methods. ${ }^{13,15}$

Approximately $30 \%-50 \%$ of adults aged 80 years or older may be adversely affected by sarcopenia. ${ }^{16}$ This condition often co-occurs with an absolute or relative increase in fat mass; this scenario, termed sarcopenic obesity ${ }^{17,18}$, may confer a cumulative risk derived from the combination of the two body composition phenotypes. The prevalence of sarcopenic obesity among the elderly is increasing with population aging ${ }^{19-21}$, and this has led to increased interest among nutritionists, geriatricians, and public health officers regarding the impact of this combined condition on physical and cardiometabolic functions.

Two scenarios may be conducive to the onset of sarcopenic obesity. First, weight gain and especially fat gain may induce sarcopenic obesity in lean subjects. Second, in morbidly obese subjects, weight loss may induce muscle loss at varying rates, leading to the onset of sarcopenic obesity consequent to prominent losses in muscle mass (Fig. 2). ${ }^{22}$ The results of previous epidemiologic studies in Korea suggest that the former may occur mainly in women, while the latter may occur more frequently in men.

\section{PATHOPHYSIOLOGY OF OBESITY IN THE ELDERLY}

The pathophysiological mechanism of elderly obesity is complex

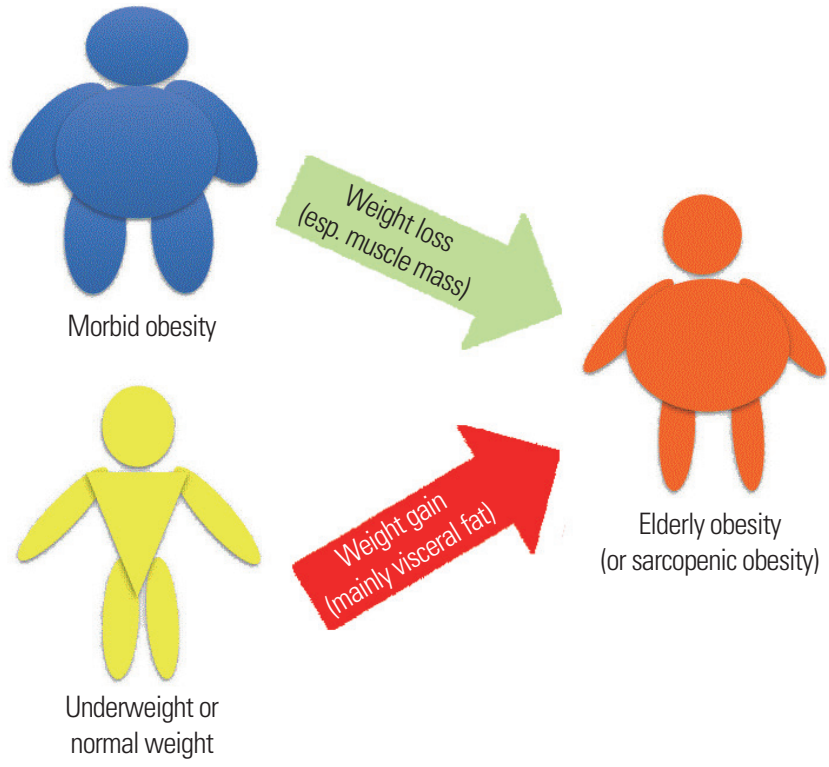

Figure 2. Two hypothetical scenarios of elderly obesity. esp, especially.

and involves interactions among aging and endocrine (insulin, leptin, corticosteroid, testosterone/estrogen, growth hormone/insulin-like growth factor [IGF]-1, vitamin D), immunological (proinflammatory cytokines, oxidative stress, mitochondrial dysfunction, apoptosis pathways) and lifestyle factors (diet, physical activity, smoking), among others. ${ }^{23,24}$ Of these, aging has been associated with important changes in body composition, such as progressive loss of skeletal muscle and increase and redistribution of body fat 
that together comprise sarcopenic obesity. ${ }^{18}$ As noted above, men begin to experience a progressive loss of muscle mass beginning at approximately 40 years of age, whereas the changes in fat distribution with age, particularly an increase in visceral fat, are more marked in women than in men. Furthermore, fat is increasingly deposited in the skeletal muscles and liver with increasing age.

In addition to ectopic fat accumulation, the loss of skeletal muscle, the largest insulin-responsive target tissue in the body, leads to insulin resistance and thus promotes a vicious cycle between muscle loss and fat gain. ${ }^{12}$ Moreover, an increase in intra-abdominal fat accumulation (i.e., visceral fat) may increase the levels of proinflammatory adipokines and oxidative stress, which further promote insulin resistance and have potentially direct catabolic effects on muscles. ${ }^{9,25,26}$ In a longitudinal KSOS study, visceral obesity was found to be independently associated with future losses of skeletal muscle mass after adjusting for confounding factors. ${ }^{27}$ This age-related progressive loss of muscle mass leads to decreases in physical performance, disability, and frailty ${ }^{24,28,29}$, which contribute to weight gain by reducing energy expenditure.

In addition to insulin resistance, obesity-related hormonal changes include decreased estrogen level in women, decreased total testosterone level in both men and women, and decreased free testosterone level in men. ${ }^{30}$ However, obesity and age-related decline in sex hormones are linked by complex relationships in the elderly. Increased adiposity is often associated with high circulating levels of free fatty acids, which inhibit growth hormone production and decrease plasma IGF-1 level. ${ }^{31}$ Therefore, low levels of or resistance to these anabolic hormones may exacerbate muscle impairment in older individuals with obesity. In addition, behavioral factors such as physical inactivity and inappropriate food intake, as well as chronic illness, can lead to muscle loss while preserving body fat. ${ }^{11}$ These multifactorial influences suggest that complex interventions may be the most effective means of counteracting obesity in the elderly.

\section{CLINICAL IMPLICATIONS OF OBESITY IN} THE ELDERLY

\section{Effects of obesity on chronic metabolic diseases}

Obesity is associated with various comorbidities, regardless of age. While obesity presents as an excess of body weight and adipose tissue in young and middle-aged adults, this condition is more physiologically and anatomically complex and less clearly quantifiable in the elderly. Although the relationship between BMI and cardiometabolic disease risk is much weaker in the elderly than in younger people, both metabolic syndrome and type 2 diabetes are positively associated with obesity indices such as BMI and waist circumference, regardless of age. ${ }^{11}$ In addition, cardiovascular risk factors such as hypertension and dyslipidemia, as well as certain cancers, are more common in older people with higher waist circumferences. $^{32,33}$

In older individuals, metabolic disorders may arise via complex mechanisms involving age-related declines in sex hormones. For example, late-onset hypogonadism in men is associated with obesity and obesity-related metabolic disorders ${ }^{34}$, while obese postmenopausal women face a greater risk of metabolic disorders relative to their lean counterparts, despite having higher total concentrations of estrogens primarily derived from the aromatization of androgens in adipose tissue. ${ }^{35}$ In one study, the chronic administration of $17 \beta$-estradiol protected against high-fat-diet-induced glucose intolerance even though exogenous estradiol can increase inflammation in adipose tissue. ${ }^{36}$ These findings suggest that androgens derived from intra-abdominal fat, rather than aromatized estrogens, may induce metabolic disorders and serve as cardiovascular risk factors.

\section{Effects of obesity on ischemic heart disease and stroke}

Excess adiposity is associated with an increased incidence of cardiovascular events. More than 9 years of follow-up data from the Korea Medical Insurance Corporation Study revealed that BMI was strongly related to the incidence of ischemic heart disease in relatively young Korean men and women (age at baseline: mean \pm standard deviation, $44.8 \pm 6.7$ years in men and $42.1 \pm 6.0$ years in women), without specific thresholds of abrupt change in risk and without a U-shaped relation. ${ }^{37}$ In addition, a heavier body weight has been associated with a modest increase in the risk of coronary heart disease in people aged 65 years. ${ }^{38,39}$ Furthermore, change in weight from middle to old age and current weight may be indicators of risk of cardiovascular disease in old age. ${ }^{40}$

In contrast, studies on the association between obesity and 
stroke in older age have yielded inconsistent results. Over a 22-year period, the Honolulu Heart Program prospectively followed a cohort of 1,163 nonsmoking men aged 55-68 years and observed a positive relationship between baseline BMI and risk of thromboembolic stroke. ${ }^{41}$ A 13-year prospective cohort study among 439,582 Korean women aged 30-95 years demonstrated that the hazard ratio of overall stroke progressively increased with increased BMI, and an increased risk of stroke was more prominent in the younger group ( $<50$ years)..$^{42}$ However, BMI was not independently associated with incidence of stroke among Korean individuals older than 50 years of age at baseline. ${ }^{42}$ A Spanish registry of 2,000 consecutive stroke patients and the Framingham Study reported that obesity was a risk factor for stroke in older women but not in older men. ${ }^{43,44}$ To further complicate the issue, a post-hoc analysis of the Systolic Hypertension in the Elderly trial identified an association of the lowest BMI quintile, rather than obesity, with an increased occurrence of stroke in both older men and women with hypertension. ${ }^{45}$ Therefore, the results of studies of the effects of weight gain on stroke in older adults should be cautiously interpreted. Although overweight and obese older adults face a higher risk of cardiovascular disease, studies also suggest a paradoxical association of obesity in the elderly with a lower cardiovascular disease mortality rate. ${ }^{7}$

Effects of obesity on arthritis, disability, frailty, dementia, and impaired quality of life

Elderly people with obesity, especially those who faced obesity earlier in life, have a greater risk of osteoarthritis of the knee; together with sarcopenia, this condition can cause disability, physical impairment, and reduced quality of life. ${ }^{11,46}$ In contrast, low muscle mass in the absence of obesity is not associated with physical disability. ${ }^{20}$ In accordance with these findings, BMI exhibits an inverse correlation with physical performance measurements in older peo$\mathrm{ple}^{47,48}$, and compared to low muscle mass, high body fat and high BMI values are more strongly associated with the risk of functional limitations in the elderly.

In older people, obesity is known as an important risk factor for frailty, which is closely associated with impaired physical function. In the Cardiovascular Health Study, individuals who developed frailty had higher body weights and were more likely to have cen- tral obesity. ${ }^{49}$ The English Longitudinal Study of Ageing identified an association of frailty with a high waist circumference, even among underweight older people, as well as a U-shaped association of BMI with frailty. ${ }^{50}$

Other studies have identified risk factors commonly associated with coronary disease, stroke, and other vascular disorders among the predictors of dementia. One meta-analysis indicated the presence of a U-shaped association between BMI and dementia $(P=$ 0.034); in other words, both obesity and underweight were associated with increased risk of dementia. ${ }^{51}$ Although the associations of frailty and dementia with BMI both yielded U-shape curves, the poor quality of life associated with obesity may also be attributable to the relationships between BMI and the aforementioned outcomes, such as chronic metabolic disease, arthritis, and physical impairment.

\section{Effects of obesity on mortality}

The negative effects of high BMI on the risk of all-cause mortality have been well established. Despite the elevated risk of cardiovascular disease among overweight or obese older adults, however, some studies on the association between BMI and mortality have suggested a paradoxical association of overweight or obesity with reduced cardiovascular disease-related mortality in this population. This phenomenon is appropriately called the "obesity paradox."7,52 In the elderly, the association between BMI and all-cause mortality yields a U-shaped curve, indicating an increased risk of death for both underweight and obese individuals. ${ }^{7}$ Nevertheless, these observational studies have often been misinterpreted to suggest that obesity is not particularly harmful in the elderly; however, in contrast, absolute mortality risk associated with increased BMI increases up to 75 years of age..$^{53}$ Notably, the obesity paradox may represent an artificial defect caused by the use of BMI to measure obesity in the elderly. Specifically, very old people with a low BMI comprise two groups: those who have always been lean and physically active and those who were often physically inactive and lost weight because of chronic illness or smoking habits. Accordingly, despite their similar BMI, these two groups of people may have very different body compositions and body fat distributions.

Because older adults tend to have higher proportions and different distributions of body and visceral fat, waist circumference or 
body composition analysis data should be considered together with BMI when evaluating the degree of obesity in the elderly. For example, Janssen et al. ${ }^{54}$ found that, in people aged 65 years and older, higher BMI values were associated with lower death rates after controlling for waist circumference; similarly, waist circumference was associated with a higher risk of death after controlling for BMI. These results suggest that BMI may be a reflection of lean mass in individuals with similar waist circumference values, whereas waist circumference is a reflection of visceral fat mass in subjects with similar BMI values. ${ }^{55}$ The optimal BMI and waist circumference targets in older adults remain to be validated in a large prospective trial, and more research is needed to evaluate this relationship.

\section{IS WEIGHT LOSS IN THE ELDERLY BENEFICIAL OR HARMFUL?}

In young or middle-aged people with obesity, weight loss is associated with many benefits and reduced risks of complications arising from fat burdens. In contrast, the effects of weight loss in the elderly remain controversial. Two scenarios, intentional and unintentional, can be used to classify weight loss in older adults, and it may be important to differentiate these scenarios when reviewing the positive and negative effects of weight loss in older people with obesity. For example, those who experience unintentional weight loss may be more likely to smoke and have chronic illnesses such as cancer or chronic heart, lung, and kidney disease. In contrast, those who experience intentional weight loss may experience clinical benefits with regard to osteoarthritis, functional disability, type 2 diabetes, and cardiovascular disease, despite slight decreases in bone mineral density and skeletal muscle mass. ${ }^{56,57}$ In addition, observational studies have demonstrated increases in life expectancy among older individuals with type 2 diabetes who lost weight intentionally. ${ }^{58}$ However, few clinical trials have addressed the association between intentional weight loss and mortality among older adults with obesity; therefore, limited evidence supports the association of weight reduction with long life expectancy.

The existing evidence suggests that intentional weight loss should be recommended specifically to older adults with obesityrelated comorbidities, including those with functional limitations or metabolic complications such as metabolic syndrome, type $2 \mathrm{di}$ abetes, and cardiovascular disease. Although the current therapeutic options for weight management in older adults do not differ from those offered to younger or middle-aged people, intentional weight loss strategies offered to older people should pay more attention to preventing loss of bone and muscle. Although older individuals are already likely to have relatively low muscle mass, further reductions due to weight loss could be mitigated by a regimen of regular resistance exercise combined with adequate protein intake. $^{59}$

\section{CONCLUSION}

The aging of the Korean population has led to an increase in the population of older adults with obesity. Obesity is a chronic disease associated with cardiovascular diseases and functional impairment, regardless of age. Therefore, aging and obesity comprise a significant health crisis in terms of the risk of sarcopenic obesity. In addition to the increased health risks, the possible mechanisms and clinical implications of obesity are more complicated and less well understood in older adults than in younger people. Therefore, more research is needed to determine the optimal BMI and waist circumference values and other potential indices of body composition in the elderly, as these will help to guide therapeutic decisions. A better understanding of the complexity of elderly obesity, from the viewpoint of sarcopenic obesity, is expected to facilitate the design of preventative and therapeutic strategies that will positively impact the overall health of the population.

\section{CONFLICTS OF INTEREST}

The author declares no conflict of interest.

\section{ACKNOWLEDGMENTS}

This research was supported by the Priority Research Centers Program through the National Research Foundation of Korea (NRF) funded by the Ministry of Education, Science and Technology (2010-0020224). 


\section{REFERENCES}

1. Molino S, Dossena M, Buonocore D, Verri M. Sarcopenic obesity: an appraisal of the current status of knowledge and management in elderly people. J Nutr Health Aging 2016;20: 780-8.

2. Lechleitner M. Obesity and the metabolic syndrome in the elderly: a mini-review. Gerontology 2008;54:253-9.

3. Kontis V, Bennett JE, Mathers CD, Li G, Foreman K, Ezzati M. Future life expectancy in 35 industrialised countries: projections with a Bayesian model ensemble. Lancet 2017;389: 1323-35.

4. Ng M, Fleming T, Robinson M, Thomson B, Graetz N, Margono $\mathrm{C}$, et al. Global, regional, and national prevalence of overweight and obesity in children and adults during 19802013: a systematic analysis for the Global Burden of Disease Study 2013. Lancet 2014;384:766-81.

5. Scully T. Public health: society at large. Nature 2014;508:S50-1. 6. Seo MH, Kim YH, Han K, Jung JH, Park YG, Lee SS, et al. Prevalence of obesity and incidence of obesity-related comorbidities in Koreans based on national health insurance service health checkup data 2006-2015. J Obes Metab Syndr 2018; 27:46-52.

7. Oreopoulos A, Kalantar-Zadeh K, Sharma AM, Fonarow GC. The obesity paradox in the elderly: potential mechanisms and clinical implications. Clin Geriatr Med 2009;25:643-59.

8. Newman AB, Lee JS, Visser M, Goodpaster BH, Kritchevsky SB, Tylavsky FA, et al. Weight change and the conservation of lean mass in old age: the Health, Aging and Body Composition Study. Am J Clin Nutr 2005;82:872-8.

9. Schrager MA, Metter EJ, Simonsick E, Ble A, Bandinelli S, Lauretani F, et al. Sarcopenic obesity and inflammation in the InCHIANTI study. J Appl Physiol (1985) 2007;102:919-25.

10. Dorner TE, Rieder A. Obesity paradox in elderly patients with cardiovascular diseases. Int J Cardiol 2012;155:56-65.

11. Han TS, Tajar A, Lean ME. Obesity and weight management in the elderly. Br Med Bull 2011;97:169-96.

12. Mathus-Vliegen EM; Obesity Management Task Force of the European Association for the Study of Obesity. Prevalence, pathophysiology, health consequences and treatment options of obesity in the elderly: a guideline. Obes Facts 2012;5:46083.

13. Kim TN, Yang SJ, Yoo HJ, Lim KI, Kang HJ, Song W, et al. Prevalence of sarcopenia and sarcopenic obesity in Korean adults: the Korean sarcopenic obesity study. Int J Obes (Lond) 2009;33:885-92.

14. Kim KM, Jang HC, Lim S. Differences among skeletal muscle mass indices derived from height-, weight-, and body mass index-adjusted models in assessing sarcopenia. Korean J Intern Med 2016;31:643-50.

15. Kim YS, Lee Y, Chung YS, Lee DJ, Joo NS, Hong D, et al. Prevalence of sarcopenia and sarcopenic obesity in the Korean population based on the Fourth Korean National Health and Nutritional Examination Surveys. J Gerontol A Biol Sci Med Sci 2012;67:1107-13.

16. Batsis JA, Mackenzie TA, Lopez-Jimenez F, Bartels SJ. Sarcopenia, sarcopenic obesity, and functional impairments in older adults: National Health and Nutrition Examination Surveys 1999-2004. Nutr Res 2015;35:1031-9.

17. Roubenoff R. Sarcopenic obesity: does muscle loss cause fat gain? Lessons from rheumatoid arthritis and osteoarthritis. Ann N Y Acad Sci 2000;904:553-7.

18. Roubenoff R. Sarcopenic obesity: the confluence of two epidemics. Obes Res 2004;12:887-8.

19. Bouchard DR, Dionne IJ, Brochu M. Sarcopenic/obesity and physical capacity in older men and women: data from the $\mathrm{Nu}$ trition as a Determinant of Successful Aging (NuAge)-the Quebec longitudinal Study. Obesity (Silver Spring) 2009;17: 2082-8.

20. Rolland Y, Lauwers-Cances V, Cristini C, Abellan van Kan G, Janssen I, Morley JE, et al. Difficulties with physical function associated with obesity, sarcopenia, and sarcopenic-obesity in community-dwelling elderly women: the EPIDOS (EPIDemiologie de l'OSteoporose) Study. Am J Clin Nutr 2009;89:1895900.

21. Baumgartner RN, Wayne SJ, Waters DL, Janssen I, Gallagher D, Morley JE. Sarcopenic obesity predicts instrumental activities of daily living disability in the elderly. Obes Res 2004;12: 1995-2004.

22. Prado CM, Wells JC, Smith SR, Stephan BC, Siervo M. Sar- 
copenic obesity: a critical appraisal of the current evidence. Clin Nutr 2012;31:583-601.

23. Walrand S, Guillet C, Salles J, Cano N, Boirie Y. Physiopathological mechanism of sarcopenia. Clin Geriatr Med 2011;27: 365-85.

24. Zamboni M, Mazzali G, Fantin F, Rossi A, Di Francesco V. Sarcopenic obesity: a new category of obesity in the elderly. Nutr Metab Cardiovasc Dis 2008;18:388-95.

25. Roubenoff $\mathrm{R}$. Catabolism of aging: is it an inflammatory process? Curr Opin Clin Nutr Metab Care 2003;6:295-9.

26. Kim TN, Choi KM. The implications of sarcopenia and sarcopenic obesity on cardiometabolic disease. J Cell Biochem 2015;116:1171-8.

27. Kim TN, Park MS, Ryu JY, Choi HY, Hong HC, Yoo HJ, et al. Impact of visceral fat on skeletal muscle mass and vice versa in a prospective cohort study: the Korean Sarcopenic Obesity Study (KSOS). PLoS One 2014;9:e115407.

28. Florez H, Troen BR. Fat and inflammaging: a dual path to unfitness in elderly people? J Am Geriatr Soc 2008;56:558-60.

29. Waters DL, Baumgartner RN, Garry PJ, Vellas B. Advantages of dietary, exercise-related, and therapeutic interventions to prevent and treat sarcopenia in adult patients: an update. Clin Interv Aging 2010;5:259-70.

30. Kennedy RL, Chokkalingham K, Srinivasan R. Obesity in the elderly: who should we be treating, and why, and how? Curr Opin Clin Nutr Metab Care 2004;7:3-9.

31. Stenholm S, Harris TB, Rantanen T, Visser M, Kritchevsky SB, Ferrucci L. Sarcopenic obesity: definition, cause and consequences. Curr Opin Clin Nutr Metab Care 2008;11:693700.

32. Iwao S, Iwao N, Muller DC, Elahi D, Shimokata H, Andres R. Effect of aging on the relationship between multiple risk factors and waist circumference. J Am Geriatr Soc 2000;48:78894.

33. Folsom AR, Kushi LH, Anderson KE, Mink PJ, Olson JE, Hong CP, et al. Associations of general and abdominal obesity with multiple health outcomes in older women: the Iowa Women's Health Study. Arch Intern Med 2000;160:2117-28.

34. Corona G, Mannucci E, Forti G, Maggi M. Hypogonadism, $\mathrm{ED}$, metabolic syndrome and obesity: a pathological link sup- porting cardiovascular diseases. Int J Androl 2009;32:587-98.

35. Cignarella A, Kratz M, Bolego C. Emerging role of estrogen in the control of cardiometabolic disease. Trends Pharmacol Sci 2010;31:183-9.

36. Riant E, Waget A, Cogo H, Arnal JF, Burcelin R, Gourdy P. Estrogens protect against high-fat diet-induced insulin resistance and glucose intolerance in mice. Endocrinology 2009; 150:2109-17.

37. Jee SH, Pastor-Barriuso R, Appel LJ, Suh I, Miller ER 3rd, Guallar E. Body mass index and incident ischemic heart disease in South Korean men and women. Am J Epidemiol 2005; 162:42-8

38. Harris T, Cook EF, Kannel WB, Goldman L. Proportional hazards analysis of risk factors for coronary heart disease in individuals aged 65 or older. J Am Geriatr Soc 1988;36:1023-8.

39. Harris TB, Ballard-Barbasch R, Madans J, Makuc DM, Feldman JJ. Overweight, weight loss, and risk of coronary heart disease in older women: The NHANES I Epidemiologic Follow-up Study. Am J Epidemiol 1993;137:1318-27.

40. Harris TB, Launer LJ, Madans J, Feldman JJ. Cohort study of effect of being overweight and change in weight on risk of coronary heart disease in old age. BMJ 1997;314:1791-4.

41. Abbott RD, Behrens GR, Sharp DS, Rodriguez BL, Burchfiel $\mathrm{CM}$, Ross GW, et al. Body mass index and thromboembolic stroke in nonsmoking men in older middle age: the Honolulu Heart Program. Stroke 1994;25:2370-6.

42. Park JW, Lee SY, Kim SY, Choe H, Jee SH. BMI and stroke risk in Korean women. Obesity (Silver Spring) 2008;16:396401.

43. Arboix A, Oliveres M, García-Eroles L, Maragall C, Massons J, Targa C. Acute cerebrovascular disease in women. Eur Neurol 2001;45:199-205.

44. Aronow WS, Ahn C, Gutstein H. Risk factors for new atherothrombotic brain infarction in 664 older men and 1,488 older women. Am J Cardiol 1996;77:1381-3.

45. Wassertheil-Smoller S, Fann C, Allman RM, Black HR, Camel GH, Davis B, et al. Relation of low body mass to death and stroke in the systolic hypertension in the elderly program. Arch Intern Med 2000;160:494-500.

46. Zamboni M, Mazzali G, Zoico E, Harris TB, Meigs JB, Di 
Francesco V, et al. Health consequences of obesity in the elderly: a review of four unresolved questions. Int J Obes (Lond) 2005;29:1011-29.

47. Jensen GL, Friedmann JM. Obesity is associated with functional decline in community-dwelling rural older persons. J Am Geriatr Soc 2002;50:918-23.

48. Launer LJ, Harris T, Rumpel C, Madans J. Body mass index, weight change, and risk of mobility disability in middle-aged and older women: the epidemiologic follow-up study of NHANES I. JAMA 1994;271:1093-8.

49. Barzilay JI, Blaum C, Moore T, Xue QL, Hirsch CH, Walston $\mathrm{JD}$, et al. Insulin resistance and inflammation as precursors of frailty: the Cardiovascular Health Study. Arch Intern Med 2007; 167:635-41.

50. Hubbard RE, Lang IA, Llewellyn DJ, Rockwood K. Frailty, body mass index, and abdominal obesity in older people. $\mathrm{J}$ Gerontol A Biol Sci Med Sci 2010;65:377-81.

51. Beydoun MA, Beydoun HA, Wang Y. Obesity and central obesity as risk factors for incident dementia and its subtypes: a systematic review and meta-analysis. Obes Rev 2008;9:20418.

52. Kalantar-Zadeh K, Horwich TB, Oreopoulos A, Kovesdy CP, Younessi H, Anker SD, et al. Risk factor paradox in wasting diseases. Curr Opin Clin Nutr Metab Care 2007;10:433-42. 53. Stevens J, Cai J, Pamuk ER, Williamson DF, Thun MJ, Wood JL. The effect of age on the association between body-mass index and mortality. N Engl J Med 1998;338:1-7.

54. Janssen I, Katzmarzyk PT, Ross R. Body mass index is inversely related to mortality in older people after adjustment for waist circumference. J Am Geriatr Soc 2005;53:2112-8.

55. Donini LM, Savina C, Gennaro E, De Felice MR, Rosano A, Pandolfo MM, et al. A systematic review of the literature concerning the relationship between obesity and mortality in the elderly. J Nutr Health Aging 2012;16:89-98.

56. Cetin DC, Nasr G. Obesity in the elderly: more complicated than you think. Cleve Clin J Med 2014;81:51-61.

57. Wannamethee SG, Shaper AG, Whincup PH, Walker M. Characteristics of older men who lose weight intentionally or unintentionally. Am J Epidemiol 2000;151:667-75.

58. Williamson DF, Thompson TJ, Thun M, Flanders D, Pamuk $\mathrm{E}$, Byers T. Intentional weight loss and mortality among overweight individuals with diabetes. Diabetes Care 2000;23:1499504.

59. Coker RH, Wolfe RR. Weight loss strategies in the elderly: a clinical conundrum. Obesity (Silver Spring) 2018;26:22-8. 